\title{
Microsaccadic eye movements and binocular rivalry
}

\author{
HOWARD W. SABRIN \\ Biomedical Engineering Center \\ Technological Institute, Northwestern University, Evanston, Illinois 60201 \\ and \\ ANDREW E. KERTESZ \\ Department of Electrical Engineering and Computer Science \\ Technological Institute, Northwestern University, Evanston, Illinois 60201
}

\begin{abstract}
Eye movements were monitored with a sensitive binocular measuring device during presentation of stimuli that caused binocular rivalry. It was found that the number of microsaccades was approximately $50 \%$ higher when measured during rivalry than when measured during periods of normal viewing. The level of microsaccadic activity is greater at the beginning than toward the end of the suppression interval. The results suggest that the depth of suppression associated with binocular rivalry is not constant over the duration of a rivalry interval.
\end{abstract}

Binocular rivalry occurs when greatly dissimilar stimuli are dichoptically presented to an observer. When the monocular stimuli are sufficiently different to be irreconcilable (i.e., they cannot be fused into a single stable percept), the subject will report alternating periods during which only one of the two stimuli are visible. Although the suppression of the nondominant stimulus during one of these periods is usually total, it has been reported (Wales \& Fox, 1970) that visual thresholds for the suppressed eye are raised by only $.5 \log$ unit.

As yet undetermined is how important a role rivalry or a similar suppressive mechanism plays in normal binocular vision, although it is considered central to the suppression theories of vision (Asher, 1953; Kaufman, 1974; Kaufman \& Arditi, 1976; Levelt, 1965; Makous \& Sanders, 1978; Verhoeff, 1935). Equally undetermined is the nature of the mechanism that mediates the perceptual alternations encountered during rivalry. Whether the disappearance of one eye's image is caused and maintained by inhibitory inputs from the dominant eye (reciprocal inhibition) or is controlled by a process independent of the other eye is the subject of ongoing research. Fox and Check (1972) presented evidence in opposition to the reciprocal inhibition model when they measured the magnitude of suppression during rivalry and found that it remained constant throughout the length of a suppression phase (the reciprocal inhibition theory

This study was supported in part by the National Institutes of Health, USPHS Grants EY 1055 and GM874, and by RCDA Grant EY70887 to A. E. Kertesz. The authors also wish to express their gratitude to Dr. Robert Fox for his advice. would predict a declining time course of suppression magnitude).

Peckham (1936) was first to study the relationship between eye movements and rivalry. He postulated that large nonconjugate eye movements would shift one image away from the fovea, causing the other stimulus to dominate. Using a relatively crude (resolution of $.5 \mathrm{deg}$ ) eye-movement measuring method, he was unable to find evidence for such movements. Kaufman (1963) suggested that nonconjugate movements cause the rivaling images to slide across each other, thus leading to what he termed the "spread of suppression."

Whittle (1963) proposed a role for conjugate movements in determining the parameters of rivalry (percentage of dominance by either stimulus, alternation frequency, etc.). He attributed the apparent preference for vertical over horizontal contours to the more frequent occurrence of horizontal eye movements, which cause a relatively greater change in the position of vertical stimuli.

Levelt $(1965,1967)$ suggested that the major role of eye movements during rivalry was one of restoring the suppressed image to a condition of dominance. $\mathrm{He}$ found that the lengths of the dominance and suppression intervals could be adequately described by a gamma distribution with 4 degrees of freedom. Fox and Herrman (1967) supported these statistical results and observed that the lengths of successive intervals were independent. Levelt concluded that four "arrivals" from a Poisson process might be necessary before a change in dominance occurred. He speculated that fixational microsaccades might serve as these "spikes of information." Microsaccades could not be the sole source of these spikes, 
since rivalry has been observed between retinally stabilized afterimages (Blake, Fox, \& McIntyre, 1971; Wade, 1973). It has been found that in the case of afterimages, the statistical distribution of the intervals (gamma, with $4 \mathrm{df}$ ) remains the same although the mean interval length increases, indicating that eye movements may well contribute toward the reaching of a threshold at which the shift in dominance occurs.

Thus, several investigators have postulated various roles for eye movements during rivalry, but all of these hypotheses are based on either subjective or theoretical considerations and thus lack objective, experimentally obtained eye-movement data.

The purpose of the current experiments was to investigate the role of microsaccadic movements during binocular rivalry by utilizing a sensitive, objective, binocular eye-movement measuring device. Our objective was to determine whether the microsaccades indeed facilitate the recovery of an image from a state of phenomenal suppression and, if so, whether the frequency of occurrence of microsaccades would yield additional information as to the nature of suppression itself. We wanted to see if the occurrence of binocular rivalry elicits a change in the rate at which microsaccades occur and, if there is such a change, whether its time course corresponds to the changing percepts associated with rivalry.

\section{METHODS}

Two right-handed males served as subjects. Each subject was seated in a dark room, with his head held stationary by a head rest and molded bite bar. Vision was corrected to 20/20.

The stimuli, shown in Figure 1, were presented dichoptically as back-illuminated transparencies. The small central circles subtending $1 \mathrm{deg}$ were the portions of the stimulus whose perceived appearance would determine whether the subject's left or right eye was dominant. The outer white ring subtended $3 \mathrm{deg}$. The luminance of the white portions of the patterns was $35 \mathrm{~cd} / \mathrm{m}^{2}$.

During presentation of rivalrous stimuli, the subject was instructed to fixate the center of the pattern and was told that he must decide whether he saw a white or a black center circle. When he saw a white circle, he was to depress a button, and when he observed a black circle, he was to release the button.

Eye movements were measured by two SRI International Double Purkinje Image Eyetrackers. Details of the eyetrackers' operation have been described by Cornsweet and Crane (1973). The output of the eyetrackers and of the subject response indicator were digitized at a rate of $120 \mathrm{~Hz}$. Each experimental run lasted $45 \mathrm{sec}$. An entire experiment consisted of 12 runs.

The eye-movement records were scanned by computer, and the positions and magnitudes of microsaccades were measured. We defined a microsaccade as an eye movement of at least 4 min of arc that occurred within a 50 -msec period.

\section{EXPERIMENT 1}

The objective of this experiment was to observe the incidence of microsaccades during binocular rivalry and to compare it with eye movements measured during normal (nonrivalrous) viewing.
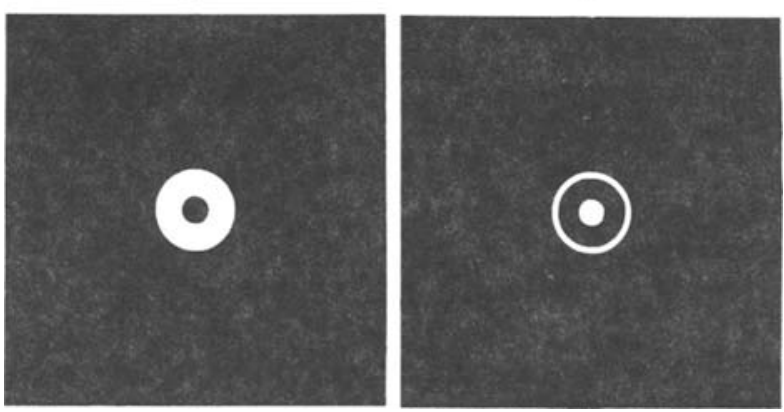

Figure 1. Stimuli used to generate binocular rivalry. Figure $1 \mathrm{~A}$ was presented to the left eye and Figure $1 B$ was shown to the right eye. The center circle subtends 1 deg of arc. The outer white ring subtends 3 deg of arc.

Microsaccades were measured during experimental runs when the subject viewed the rivalrous stimuli shown in Figure 1 and during runs when the pattern with the black center (Figure 1A) was presented to both eyes. In this experiment, the subject was not required to indicate the current state of dominance during rivalrous presentation. Afterwards, however, both subjects reported that the phenomenal suppression was always complete (i.e., the central circle always appeared either totally black or totally white).

The results of Experiment 1 are presented in Table 1. The rate of microsaccadic activity increased by about $50 \%$ for both subjects during rivalrous stimulation. This increase was found to be statistically significant using the chi-square one-sample test at the .01 level of significance. The next logical question is whether the increased oculomotor activity observed during rivalry is constant over the rivalry periods or, if not, what relationship exists between the change in activity and the length of rivalry intervals.

\section{EXPERIMENT 2}

In this experiment, we observed the distribution of microsaccades within individual intervals of dominance and suppression. The subject reported the current state of dominance. Periods of left- and right-eye dominance were analyzed separately. To compensate for the variation in the lengths of the intervals (which normally ran from $1-8 \mathrm{sec}$ ), the interval lengths were normalized and the relative positions of microsaccades within the intervals were recorded. The results are shown in Figures 2 and 3.

Table 1

\begin{tabular}{cccc} 
& \multicolumn{2}{c}{ Number of Saccades per Minute } & \\
\cline { 2 - 3 } Subject & $\begin{array}{r}\text { Normal } \\
\text { Viewing }\end{array}$ & $\begin{array}{c}\text { Rivalrous } \\
\text { Viewing }\end{array}$ & $\begin{array}{c}\text { Percent } \\
\text { Increase }\end{array}$ \\
\hline A.L.P. & 78.4 & 116.9 & 49.1 \\
H.W.S. & 86.0 & 135.3 & 57.3 \\
\hline
\end{tabular}



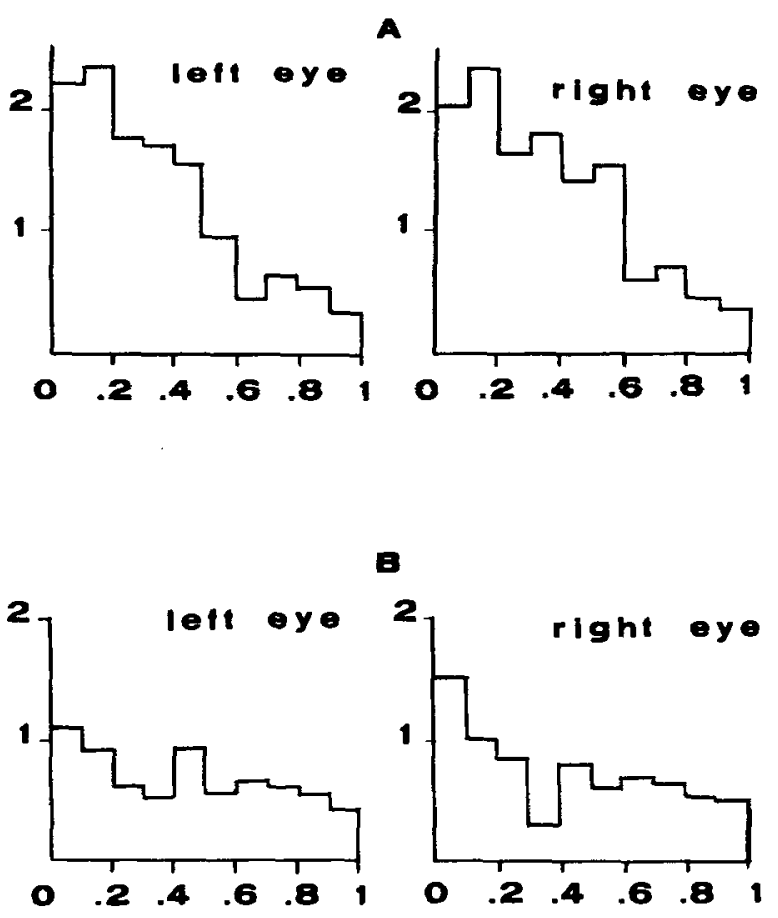

Figure 2. (A) Number of microsaccades vs. normalized interval duration for periods of right-eye dominance. Subject A.L.P. (B) Number of microsaccades vs. normalized interval duration for periods of left-eye dominance. Subject A.L.P.

We note that, for both subjects, during periods of right-eye dominance (Figures $2 \mathrm{~A}$ and $3 \mathrm{~A}$ ), an initially high rate of microsaccadic activity is followed by a rapid falloff which eventually reaches the activity level seen under nonrivalrous viewing conditions. The responses associated with left-eye dominance are shown in Figures 2B and 3B. Here, the responses are relatively flat, with the saccade rate only slightly above that for normal viewing. To determine whether the differences between microsaccade distributions for the left and right eyes are statistically significant, Kolmogorov-Smirnov two-sample tests were performed. The results showed (at the .01 significant level) that the microsaccade distributions seen in Figures 2B and 3B are different from those seen in Figures $2 \mathrm{~A}$ and $3 \mathrm{~A}$, respectively.

A control experiment was performed to ensure that eye movements did not occur as an artifact of depressing and releasing the subject's response button. Subject H.W.S. depressed and released the response button randomly while viewing nonrivalrous stimuli. The results, displayed in Figure 4 (for the left eye only) show a flat response, leading us to conclude that any change in the eye-movement pattern under rivalrous viewing conditions is indeed caused by the rivalry itself.

\section{EXPERIMENT 3}

Experiment 3 was performed to determine whether the microsaccade profiles obtained in Experiment 2
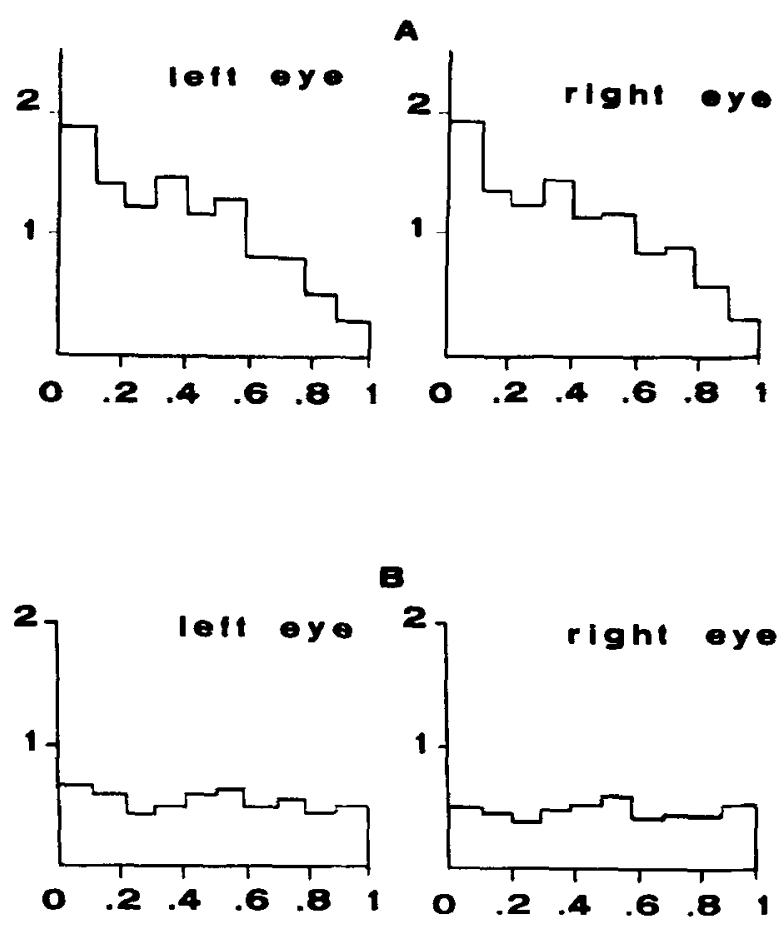

Figure 3. (A) Number of microsaccades vs. normalized interval duration for periods of right-eye dominance. Subject H.W.S. (B) Number of microsaccades vs. normalized interval duration for periods of left-eye dominance. Subject H.W.S.

were caused by the dominant or the suppressed eye. The stimuli used are shown in Figure $1 B$ and were displayed on oscilloscopes which were controlled by an LSI-11 computer.

The experiment consisted of alternating periods of stimulus presentation. During one presentation, both eyes were shown the pattern seen in Figure 1B. During the other presentation, one eye saw the same stimulus, while the other eye viewed a pattern from which the central circle was missing. During the latter interval, the central circle was still perceived, since it would always dominate over a blank field. Each interval lasted $5 \mathrm{sec}$ and each experimental run lasted $40 \mathrm{sec}$. The subject was not required to depress a button to indicate his percept, as the computer monitored the stimulus conditions.
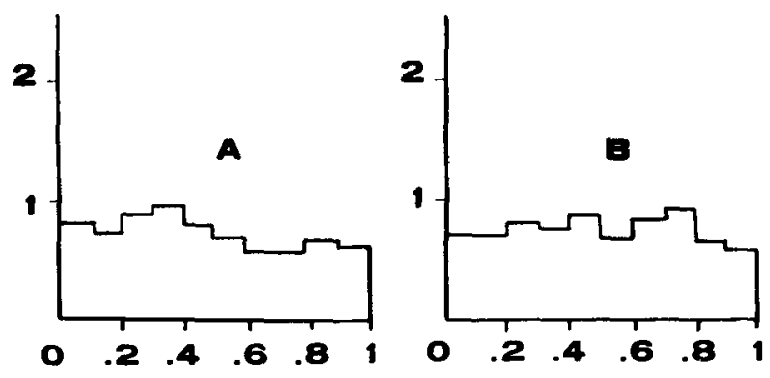

Figure 4. Number of microsaccades of the left eye vs. normalized interval duration measured during nonrivalrous stimulus presentation. Subject depressed and released button at random. (A) Button depressed. (B) Button released. Subject H.W.S. 
The purpose of this experiment was to create a condition whereby one eye always remained in a dominant state (its stimulus was always observed), while the other eye alternated between states analogous to dominance and suppression. In this way, we have altered the conditions of Experiment 2, in which each onset of suppression for one eye is accompanied by a simultaneous onset of dominance for the other eye. By doing so, we can see whether the falloff in the number of microsaccades observed during a dominance-suppression interval in Experiment 2 is caused by the eye undergoing dominance or by the eye that is in a suppressed state.

The results of Experiment 3 (for Subject A.L.P.) are seen in Figures 5 and 6 . Figure 5 shows the experimental results for the case when the left eye is shown the disappearing stimulus, while Figure 6 contains the results corresponding to the case when the central circle shown the right eye disappears. In Figures $5 \mathrm{~B}$ and $6 \mathrm{~A}$, we see that during periods when both eyes receive identical stimulation (the central circle is displayed on both oscilloscopes), the distribution of microsaccades across the interval is relatively flat. Figure 5A gives the microsaccade distributions during intervals in which the left stimulus's central circle has disappeared. Here, we see the same characteristic drop-off in the microsaccade rate that we saw in Experiment 2. The physical disappearance of part of the stimulus to the left eye yielded results similar to those obtained when the left eye undergoes the suppression associated with binocular rivalry. This observation is supported statistically by the KolmogorovSmirnov two-sample test, in which the data in Figure $5 \mathrm{~A}$ were found (at the .01 significance level) to come from a distribution different from the data in Figure 5B. The data in Figure 5A were also shown to be similar in distribution to the data in Figure 2A, which represent Subject A.L.P.'s response to rivalrous stimuli while his right eye was dominant. In Figure 6B, we see that when the right central circle disappears, the microsaccade profiles remain flat. These results are consistent with the findings of Experiment 2. The Kolmogorov-Smirnov test supports the hypothesis that the results shown in Figures $6 \mathrm{~A}$ and $6 \mathrm{~B}$ have the same underlying distribution.

The results of Experiment 3 lead us to believe that the microsaccadic response observed during binocular rivalry is caused by the eye that is being suppressed and that this response is similar to that elicited by the physical removal of the stimulus.

\section{DISCUSSION}

How can we explain the interesting distribution of microsaccades during periods of right-eye dominance? Microsaccades are often associated with corrective eye movements (i.e., movements that "lock" a fixational target onto the fovea). In light of this,
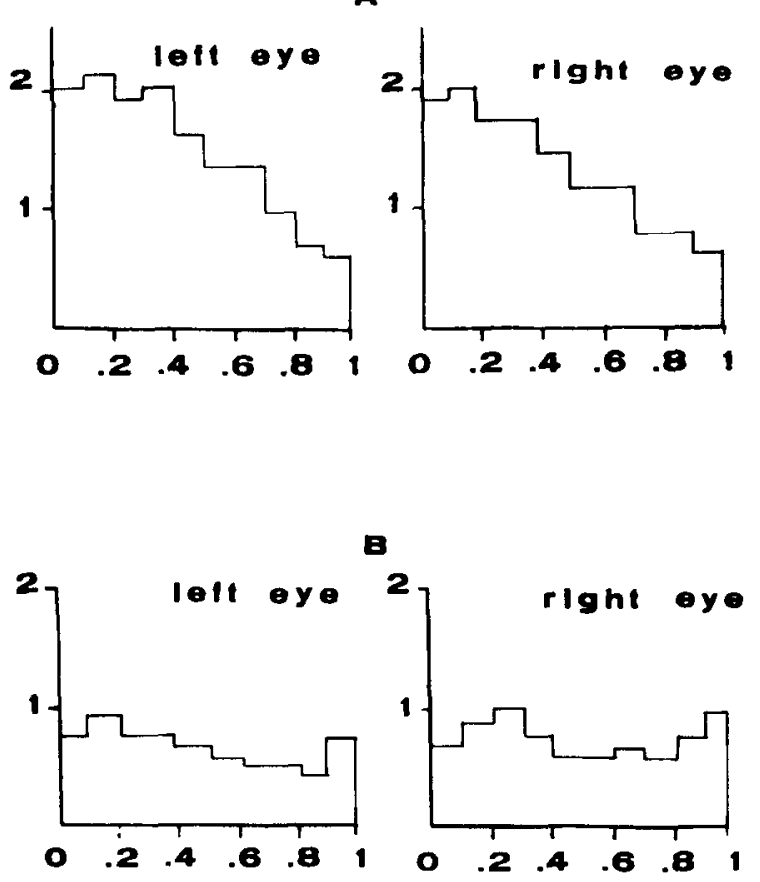

Figure 5. Results of Experiment 3 for runs in which the left eye received the alternating stimulus. (A) Number of microsaccades vs. normalized interval duration for periods when the left central circle is missing. (B) Number of microsaccades vs. normalized interval duration for periods when left and right stimuli are identical. Subject A.L.P.
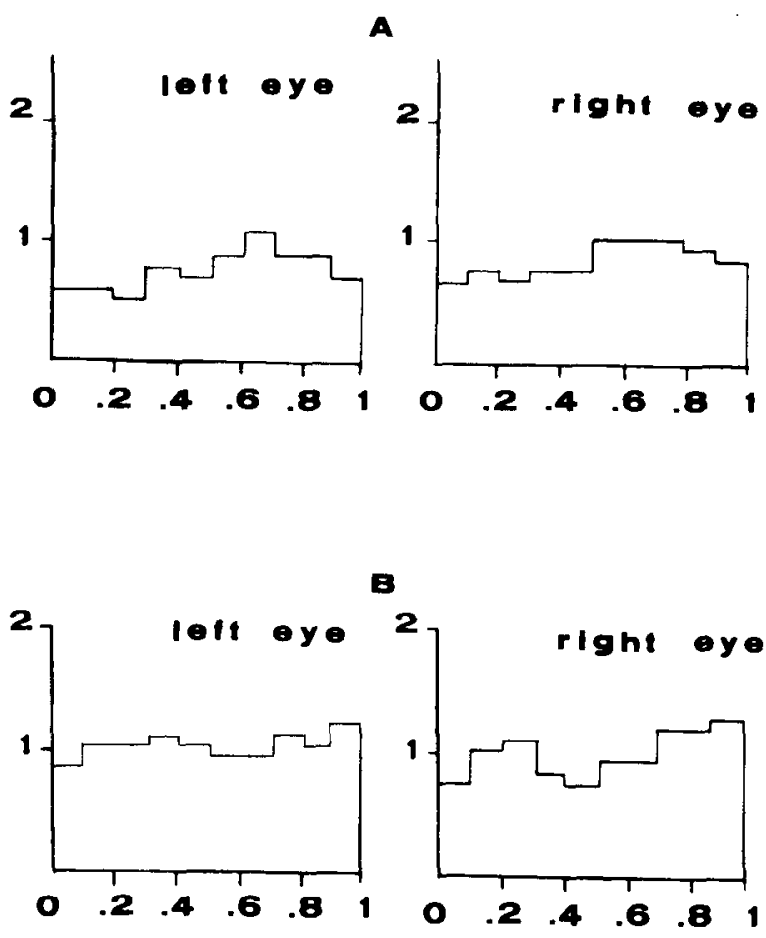

Figure 6. Results of Experiment 3 for runs in which the right eye received the alternating stimulus. (A) Number of microsaccades vs. normalized interval duration for periods when left and right stimuli are identical. (B) Number of microsaccades vs. normalized interval duration for periods when the right central circle is missing. Subject A.L.P. 
it is reasonable to suggest that the oculomotor system might respond with an increased microsaccadic activity to the perceptual loss of a fixational target caused by the suppression associated with binocular rivalry. At the onset of suppression, when the image is suddenly lost, we might, therefore, see a high rate of microsaccadic activity. As the interval wears on, and information concerning the lost stimulus is accumulated at some neural level, the signal to the oculomotor system that caused the increased activity would decline. This could be interpreted as representing a decline in the magnitude of suppression. This interpretation of our results is in conflict with that of Fox and Check (1972), who, as previously mentioned, found that the suppression magnitude remained at a constant level throughout the interval. One possible explanation of this difference is that Fox and Check's data were not separated into periods of left- and right-eye dominance. Our data suggest significant differences between the responses during the leftand right-dominance intervals, perhaps caused by eye dominance or by different "gains" in the mechanisms mediating the oculomotor response to suppression in the two eyes. Therefore, pooling the binocular data for intervals of left and right dominance might obscure the results. Another possible reason for the dissimilarity of our results is that, while at the conscious level the suppression associated with rivalry is all or none, there may exist neural centers where information about the suppressed image is gradually retrieved or perhaps is not lost at all. Thus, measurements requiring a visual recognition of a test probe (i.e., visual threshold measurements) might not reflect the true course of the suppression level that mediates the return to dominance of the suppressed image. This interpretation would be consistent with the results of Kaufman (1974), who found that the suppressed image in a rivalrous presentation can contribute toward the perception of depth, and with those of Piantanida and Crane (Note 1), who found that the same is true for a faded, retinally stabilized image.

\section{SUMMARY}

We found that the rate of occurrence of microsaccades significantly increases during binocular rivalry. During periods of right-eye dominance, an initially high rate of microsaccadic activity is followed by a decreasing rate that eventually reaches the level measured during normal viewing. This suggests that the level of suppression is not constant over a suppression interval.

The observed increase in microsaccadic activity and the distribution of microsaccades over a suppression interval lend support to Levelt's $(1965,1967)$ hypothesis that microsaccadic eye movements play a role in the determination of alternation times during rivalry.

\section{REFERENCE NOTE}

1. Piantanida, T. P., \& Crane, H. D. Selective stabilization of retinal images. Paper presented to the Association for Research in Vision and Ophthalmology, 1979.

\section{REFERENCES}

Asher, H. Suppression theory of binocular vision. British Journal of Ophthalmology, 1953, 37, 37-39.

Blake, R., Fox, R., \& McInTYRe, C. Stochastic properties of stabilized image binocular rivalry alternations. Journal of Experimental Psychology, 1971, 88, 327-332.

Cornsweet, T. N., \& Crane, H. D. Accurate two-dimensional eyetracker using first and fourth Purkinje images. Journal of the Optical Society of America, 1973, 63, 921-928.

Fox, R., \& Check, R. Independence between binocular rivalry suppression duration and magnitude of suppression. Journal of Experimental Psychology, 1972, 93, 283-289.

Fox, R., \& Herhman, J. Stochastic properties of binocular rivalry alternations. Perception \& Psychophysics, 1967, 2, 432-436.

KaUFMAN, $L$. On the spread of suppression and binocular rivalry. Vision Research, 1963,3, 401-415.

Kaufman, L. Sight \& Mind: An introduction to visual perception. New York: Oxford University Press, 1974.

Kaufman, L., \& Arditi, A. The fusion illusion. Vision Research, $1976,16,535-544$.

Levelt, W. On binocular rivalry. Soesterberg, The Netherlands: Institute for Perception RVO/TNO, 1965.

TEVELT, $W$. Note on the distribution of dominance times in binocular rivalry. British Journal of Psychology, 1967, 58, 143-145.

Makous, W., \& Sanders, R. K. Suppressive interactions between fused patterns. In A. C. Armington, J. Krauskopf, \& B. R. Wooten (Eds.), Visual psychophysics and physiology. New York: Academic Press, 1978.

Peскнам, R. H. Eye movements during retinal rivalry. American Journal of Psychology, 1936, 48, 43-63.

Verhoeff, F. H. New theory of binocular vision. Archives of Ophthalmology (Chicago), 1935, 13, 151-175.

WADE, N. J. Binocular rivalry and binocular fusion of afterimages. Vision Research, 1973, 13, 999-1000.

Wales, R., \& Fox, R. Increment detection thresholds during binocular rivalry suppression. Perception \& Psychophysics, $1970,8,90-94$.

Whitrte, P. Binocular rivalry. Unpublished doctoral dissertation, Cambridge University, 1963.

(Received for publication June 18, 1979; revision accepted May 13, 1980.) 